Laser Chem., 1999, Vol. 19, pp. 51-56

Reprints available directly from the publisher Photocopying permitted by license only
(C) 1999 OPA (Overseas Publishers Association) N.V.

Published by license under

the Harwood Academic Publishers imprint,

part of The Gordon and Breach Publishing Group.

Printed in India.

\title{
SOLVENT-DEPENDENT RESONANCE RAMAN EXCITATION PROFILES OF 9,9'-BIANTHRYL
}

\author{
GREGORY D. SCHOLES ${ }^{\mathrm{a}}$, THIERRY FOURNIER ${ }^{\mathrm{b}}$, \\ DAVID PHILLIPS $^{\mathrm{a}, *}$ and ANTHONY W. PARKER ${ }^{\mathrm{b}}$ \\ a Department of Chemistry, Imperial College of Science, Technology and \\ Medicine, Exhibition Road, London SW7 2 AY, United Kingdom; \\ ${ }^{\mathrm{b}}$ Lasers for Sciences Facility, Central Laser Facility, Rutherford Appleton \\ Laboratory, Chilton, Didcot, Oxon OX11 0QX, United Kingdom
}

(Received 6 May 1997)

\begin{abstract}
Dynamics subsequent to the electronic excitation of $9,9^{\prime}$-bianthryl produce a polar emissive excited state for which the symmetry of the bichromophore is broken by a dynamic solvent stabilisation of one of the two, otherwise degenerate, charge transfer configurations which contribute to the excited electronic state. The initial state created upon excitation is examined here by analysis of ground state resonance Raman excitation profiles (REPs) and electronic absorption spectra in solvents of various polarities. The results suggest that the REPs are signalling electronic differences between the initially excited state in the various solvents. We suggest that this is related to the bianthryl excited state being responsive to the disordered solvent environment.
\end{abstract}

Keywords: Resonance Raman; molecular dynamics; bianthryl

9,9'-Bianthryl (BA) is a molecule having photophysical properties typical of a broad class of compounds which are thought to exhibit twisted intramolecular charge transfer (TICT) [1-3]. The absorption spectrum of BA is essentially solvent-independent, whereas its fluorescence emission is highly solvent-dependent owing to the charge-transfer (CT) character of the emissive state. The observation of a polar emissive state implies that the symmetry of this

*Corresponding author. e-mail: d.phillips@ic.ac.uk 
bichromophore is broken by a dynamic solvent stabilisation of one of the two, otherwise degenerate, charge transfer configurations which contribute to the excited electronic state. (This is not observed, for example, for the anthracene excimer because in this case the CT configurations are coupled strongly to the exciton configuration).

The dynamics of charge separation in the $S_{1}$ state of BA have been studied in some detail [4-9], leading to the primary conclusions that: there may be a small barrier to the charge separation; the dynamics are correlated strongly with solvent relaxation dynamics; the reaction coordinate is principally the solvent coordinate; and the electron transfer is electronically adiabatic. It is unclear whether or not vibrational degrees of freedom of the anthracene rings contribute a significant role to the dynamics. We shall address this point in the present work .

We report the preliminary results of a study of the initially excited singlet state of BA. We aim to elucidate how the dynamic Stokes' shift is initiated and to uncover any role played by intramolecular reorganisation. We are currently elucidating methods to investigate these effects by analysis of ground state resonance Raman excitation profiles (REPs) and electronic absorption spectra of BA in solvents of various polarities, since dynamical information is contained in the intensities of the resonance Raman bands owing to the effect of population of the resonant intermediate state; which may be long compared to the time-scale of nuclear motion.

The BA was synthesised and purified using previously published methods [13]. Samples were made up to concentrations of ca. 0.5 to $1 \mathrm{mM}$ (and to ca. $10^{-5} \mathrm{M}$ for the absorption spectra) in spectroscopic grade solvents (Aldrich): carbon tetrachloride $\left(\mathrm{CCI}_{4}\right)$, dichloromethane (DCM) and acetonitrile (MeCN). They were degassed by argon bubbling and flowed through a Spectrosil B quartz silica tube. A pulsed $(10 \mathrm{~Hz})$ excimer-pumped dye laser was employed as the excitation source, providing approximately $0.2 \mathrm{~mJ}$ of energy at the sample. The Raman signal was detected using a back-scattering method (to minimise reabsorption effects) at right angles to the sample flow direction with a Spex Triplemate 1877 spectrograph and a back illuminated liquid nitrogen cooled CCD detector (Princeton Instruments CSMALN/CCD-1024/TKB/I system). Typically, $90 \times 20 \mathrm{~s}$ exposures were collected. 
We report here a preliminary, qualitative analysis of the REPs using transform theory $[11,14]$. Such an analysis can provide approximate excited state displacements for each vibrational mode, which give information on the mode-specific intramolecular reorganisation energy $[15,16]$. The model involves displaced, separable harmonic oscillators with identical ground and excited state frequencies and no Duschinsky rotation. Considering the strong excitonic coupling in the excited state of BA (i.e., delocalised excitation), it is probable that these assumptions are a reasonable first approximation, although we are currently examining this further. It is also assumed that there is no inhomogenous line broadening and no coordinate dependence of the transition moment. In addition, we have employed the time-dependent analysis described by Myers et al. [15, 16].

The absorption spectra of BA in solvents of various polarities have very similar profiles, but exhibit small solvent shifts of the $0-0$ energy gaps $\left(25400 \mathrm{~cm}^{-1}\right.$ in $\mathrm{CCl}_{4}, 25500 \mathrm{~cm}^{-1}$ in $\mathrm{DCM}$ and $25650 \mathrm{~cm}^{-1}$ in $\mathrm{MeCN}$ ). In Figure 1 the resonance Raman spectra of BA (exciting at $338 \mathrm{~nm}$ ) in various solvents are compared. There are differences clearly evident in these spectra which are not apparently distinguishable in the

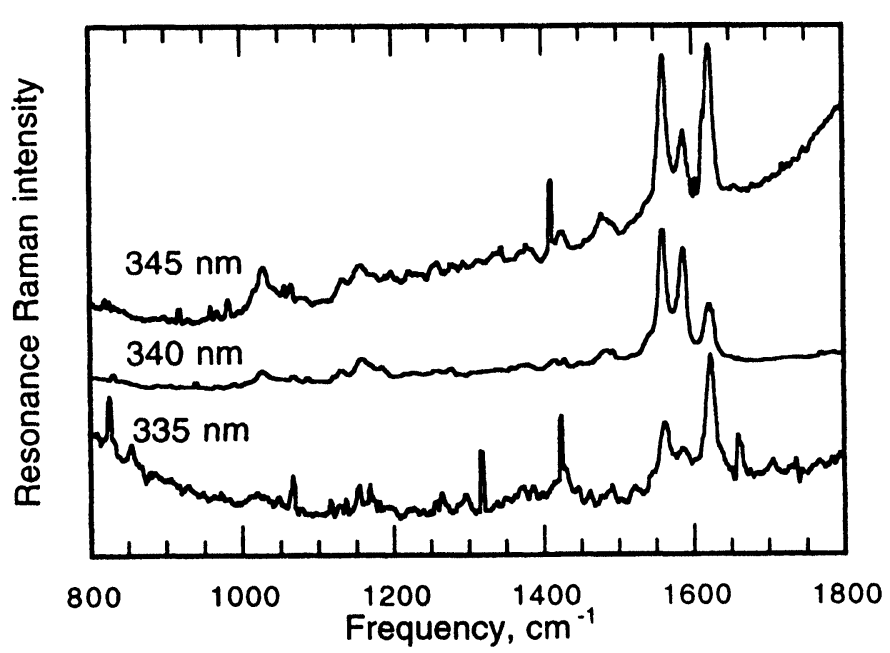

FIGURE 1 Resonance Raman spectra of 9,9'-bianthryl (excitation wavelength $338 \mathrm{~nm})$ in various solvents: carbon tetrachloride $\left(\mathrm{CCl}_{4}\right)$, dichloromethane $(\mathrm{DCM})$ and acetonitrile $(\mathrm{MeCN})$. 
absorption spectra. We used resonance Raman excitation profiles (REPs) for BA in each solvent, obtained from a series of spectra such as those depicted in Figure 2, to obtain some insight into the reasons underlying this observation.

The transform theory analysis (shown in Fig. 3 for some modes) is qualitatively useful, but fails to account properly for intensity changes within the intense $1500-1600 \mathrm{~cm}^{-1}$ region (ring modes). A timedependent analysis this region was more successful, even with the assumption of equal ground and excited state frequencies [17]. A contributing factor may be the different description of solute-bath interactions used in each theory. The transform theory is based on the assumption that the bath fluctuations are very fast compared to the time-scale of the experiment, whereas the time-dependent analysis employs a more flexible Brownian oscillator model for incorporating homogenous line broadening [18]. However, a key factor should be that the time-dependent analysis allows a vibrational wavepacket to propagate on the excited (intermediate) state surface.

We postulate that there may be solvent-related effects occurring on the time-scale of absorption which govern the subsequent dynamics. These effects would be due mainly to the disorder (inhomogeneity) of

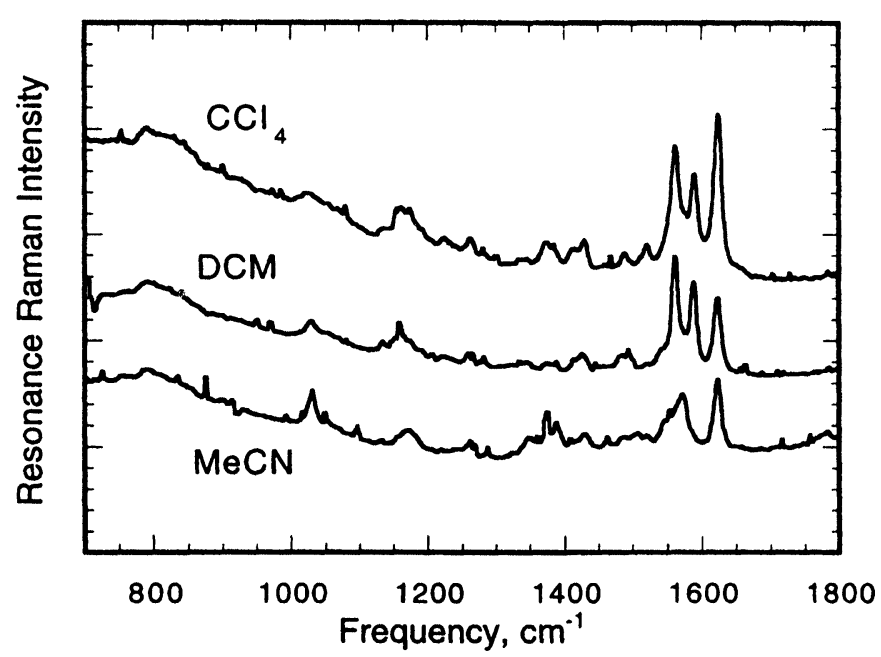

FIGURE 2 Resonance Raman spectra of BA in DCM solvent at the excitation wavelengths indicated. 


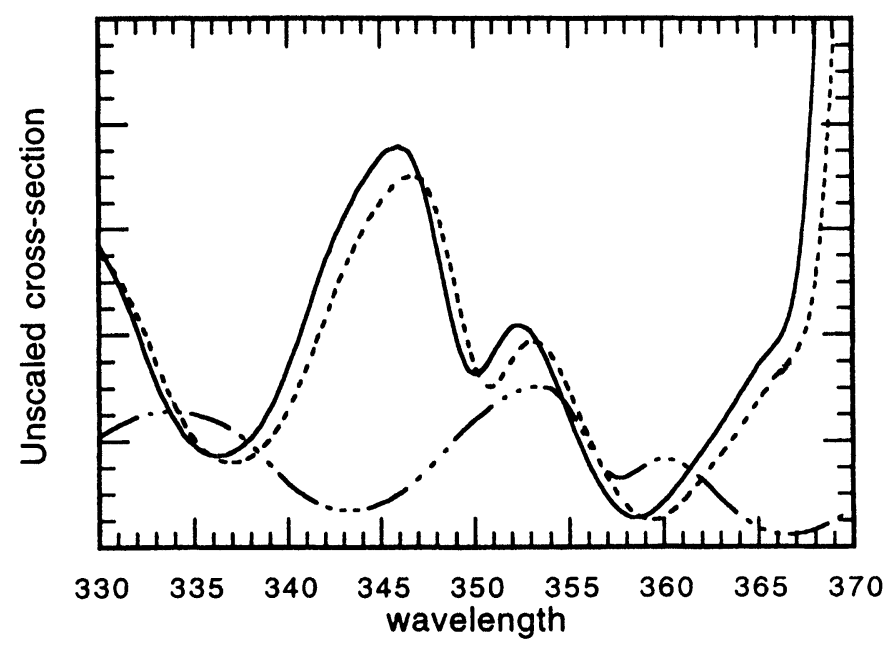

FIGURE 3 Unscaled resonance Raman cross-sections for BA in DCM solvent for the modes with frequencies $1624 \mathrm{~cm}^{-1}$ (solid line), $1562 \mathrm{~cm}^{-1}$ (dashed line) and $1016 \mathrm{~cm}^{-1}$ (mixed dashes).

the solvation shell about the BA, from which the impetus for preferential stabilisation of one of the CT configuration would arise naturally. An analogous "dynamic disorder" would arise owing to fluctuations of the environment on the time-scale of the experiment (i.e., an homogenous contribution). As a result of this disorder, some initial CT character would be present in the ensemble-average BA excited state. This may be detectable as changes in the displacements of some vibrational modes owing to changes induced in the reorganisation energy. (We refer here to the reorganisation energy required for the initial, "instantaneous" Stokes' shift, not that for the overall charge separation reaction).

The results of this study suggest that the REPs are signalling some differences between the initial dynamic response of the excited singlet state of BA to each solvent. It is quite likely that the origin of this observation is connected with the sensitivity of the BA excited state to probe the disordered solvent environment. This is being studied further using the time-dependent analysis of REPs as well as simulations of the effect of a disordered solvent environment on the nature of the bichromophore's initially excited state. 


\section{Acknowledgements}

Ms Sue Tavender is gratefully acknowledged for her valuable contributions to the resonance Raman measurements, carried out at the Central Laser Facility, Rutherford-Appleton Laboratories. Dr. P. A. Koutentis is thanked for his careful synthesis of the BA. The EPSRC is gratefully acknowledged for financial support. GDS gratefully acknowledges the support of the Ramsay Memorial Fellowship Trust.

\section{References}

[1] Rettig, W. and Zander, M. (1983). Ber Bunsenges. Phys. Chem., 87, 1143.

[2] Rettig, W. (1994). Top. Curr. Chem., 169, 254.

[3] Rettig, W. (1986). Angew. chem. Int. Ed. Engl., 25, 971.

[4] Mataga, N., Yao, H., Okada, T. and Rettig, W. (1989). J. Phys. Chem., 93, 3383.

[5] Mataga, N., Nishikawa, S. and Okada, T. (1996). Chem. Phys. Lett., 257, 327.

[6] Elich, K., Kitazawa, M., Okada, T. and Wortmann, R. (1997). J. Phys. Chem. A, 101, 20210.

[7] Kahlow, M. A., Kang, T. J. and Barbara, P. F. (1987). J. Phys. Chem., 91, 6452.

[8] Kang, T. J., Kahlow, M. A., Giser, D., Swallen, S., Nagarajan, V., Jarzeba, W. and Barbara, P. F. (1988). J. Phys. Chem., 92, 6800.

[9] Kang, T. J., Jarzeba, W., Barbara, P. F. and Fonseca, T. (1990). Chem. Phys., 149, 81.

[10] Lee, S.-Y. and Heller, E. J. (1979). J. Chem. Phys., 71, 4777.

[11] Myers, A. B. and Mathies, R. A. (1987). In: Biological Applications of Raman Spectroscopy, Ed. Spiro, T. G., Wiley, New York, Vol. 2, 1.

[12] Myers, A. B. (1990). J. Opt. Soc. Am. B, 7, 1665.

[13] Bell, F. and Waring, D. H. (1949). J. Chem. Soc., 149, 267.

[14] Tonks, D. L. and Page, J. B. (1979). Chem. Phys. Lett., 66, 449.

[15] Markel, F., Ferris, N. S., Gould, I. R. and Myers, A. B. (1992). J. Am. Chem. Soc., 114, 6208.

[16] Myers, A. B. (1994). Chem. Phys., 180, 215.

[17] Scholes, G. D., Fournier, T., Phillips, D. and Parker, A. W. (unpublished).

[18] Mukamel, S. (1995). Principles of Non-linear Optical Spectroscopy, OUP, New York. 